\title{
ESTUDOS SÔBRE A ALIMENTAÇĀO MINERAL DA MANDIOCA (Manihot utilissima Pohl.)
}

\author{
E. MALAVOLTA (1) - J. A. DE C. PACHECO (3) \\ E. A. GRANER (2) - T. COURY (1) \\ M. O. C. DO BRASIL SOBR. (1)
}

\begin{abstract}
INDICE

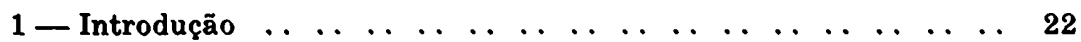

2 - Material e Métodos . . . . . . . . . . . . . . . . . . . . 22

3-Resultados e Discussão . . . . . . . . . . . . . . . . . . . . . 24

4 -Resumo e Conclusões . . . . . . . . . . . . . . . . . . . . . 36

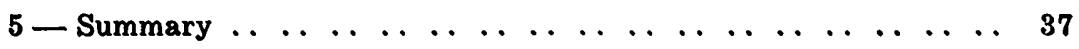

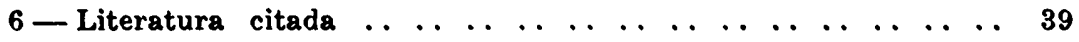

(1) Seç̧ão de Química Agrícola, E. S. A. "Luiz de Queiroz", Univ. de S. Paulo, Piracicaba, Estado de S. Paulo, Brasil.

(2) Seção de Fitotecnia, E. S. A. "Luiz de Queiroz", Univ. de S. Paulo, Piracicaba, Estado de São Paulo, Brasil.

(3) Seção de Tecnologia Agrícola, Instituto Agronômico do Estado de S. Paulo, Campinas, Estado de São Paulo, Brasil. 


\section{1 - INTRODUÇÄO}

Embora a mandioca apresente grande importância econômica para o Brasil, não só como alimento, mas também como matéria prima para produtos de exportação, não são muito numerosos os estudos sôbre a adubaçäo dessa cultura feitos em nosso meio (ver GROSSMAN, 1946, NORMANHA e PEREIRA, 1950). Os experimentos relatados, tendo sido em geral conduzidos em condições naturais de campo, não fornecem informaçōes a respeito das exigências minerais dessa euforbiácea.

Por essa razão, a E. S. A. "Luiz de Queiroz", U. S. P., em colaboração com o Instituto Agronômico do Estado de S. Paulo, resolveu iniciar uma série de ensaios que permitissem entender melhor o problema, a fim de que, conselhos mais seguros sôbre a adubação dessa planta possam ser dados. Por outro lado, deseja-se também saber o efeito da adubaçäo na quantidade e na qualidade do amido. Como o solo é um meio demasiadamente complexo, resolveu-se fazer, preliminarmente, uma cultura de mandioca, em vasos com areia lavada, à qual foram adicionados os diversos tratamentos estudados.

Uma nota prévia contendo apenas os dados culturais foi discutida na IV Reunião da Soc. Brasileira de Ciência do Solo e será publicada em outro lugar (MALAVOLTA et al., 1953).

\section{2 - MATERIAL E METODOS}

2.1. O cultivo das plantas. O ensaio foi feito em vasos de barro vidrado e esmaltado, com capacidade para 60 quilos de areia. Esta sofreu prèviamente lavagem com ácido cloridrico concentrado e depois com água de torneira e água destilada, até eliminação da acidez. Tomou-se por base, na adubação, a quantidade e a proporção de nutrientes aconselhadas por Mitscherlich (GOMES e MALAVOLTA, 1949).

Os tratamentos foram os seguintes:
N1 P1 K1
NO P1 K1
N1 P0 K1
N1 P1 K0
N2 P1 K1
N1 P2 K1
N1 P1 K2
No PO KO 
Cada tratamento, com exceção do NO P0 K0 (apenas um vaso) foi repetido quatro vêzes. As doses foram as seguintes : $\mathrm{N} 1=35$ gramas de nitrato de amônio, por vaso; $\mathrm{P} 1=35$ gramas de fosfato monocálcico por vaso e $\mathrm{K} 1=28$ gramas de sulfato de potássio por vaso; êsses macronutrientes foram adicionados como droga pura; além disso, todos os vasos receberam: 26 gramas de sulfato de magnésio e micronutrientes em doses semanais, na forma e proporções indicadas por HOAGLAND e ARNON (1939). Os vasos receberam irrigação, sempre que necessário. $O$ excesso de solução que, por vêzes atravessava a areia, era recebido em coletor de barro vidrado e esmaltado e a seguir devolvido ao vaso.

Cada vaso recebeu uma estaca de mandioca da variedade "Branca de Sta. Catarina", de $15 \mathrm{~cm}$ de comprimento, a qual foi plantada horizontalmente, em 15 de Julho de 1952. A colheita se deu em 15 de Maio de 1953. Com o fim de verificar o desenvolvimento e produção de mandioca à custa exclusivamente das reservas da maniva, incluiu-se o tratamento N0 P0 K0, sem repetição, em areia lavada, só tratada com água.

\subsection{A análise das raizes.}

2.2.1. Amostragem. Após a colheita, as raízes foram desprovidas das radicelas e do cordão lenhoso de união com a maniva original. Para as análises foi retirada, de cada vaso, uma amostra de aproximadamente $500 \mathrm{~g}$ composta de pedaços provenientes da ponta, meio e base das raízes.

2.2.2. Preparo das amostras. As amostras obtidas, picadas em fatias delgadas, foram postas para secar ao sol até se apresentarem quebradiças (12 por cento de umidade, mais ou menos). Depois da secagem, foram moidas, peneiradas e acondicionadas em frascos de vidro hermèticamente fechados.

\subsubsection{Métodos analiticos.}

2.2.3. A. Umidade e matéria sêca. Cinco gramas de amostra foram postas a secar em estufa a $100-110^{\circ} \mathrm{C}$ até pêso constante. Como a perda de umidade durante a secagem das amostras ao sol fôra anotada, foi possível determinar assim a umidade to. tal das raízes, e, por diferença, a matéria sêca. 
2.2.3. B. Amido. Foi determinado pelo método polarimétrico de EWERS (1908). O valor adotado para a rotação específica, alfa $\underset{D}{20}$, do amido de mandioca foi de 183,1, segundo RIECHELMANN (1921) citado por WALTON (1928).

2.2.3. C. Matéria mineral. Foi dosada por incineração de $5 \mathrm{~g}$ da amostra em mufla a $500^{\circ} \mathrm{C}$, pesando-se depois o resíduo incombustível.

2.2.3. D. Matéria azotada. Foi usado o método de KJELDAHL (1883), empregando-se cristais de sulfato de cobre para acelerar a reação.

\section{3 - RESULTADOS E DISCUSSÃO}

3.1. Resultados culturais. Acham-se resumidos no Quadro1.

$$
\text { QUADRO } 1
$$

Colheita obtida (em gramas)

\begin{tabular}{|c|c|c|c|c|c|}
\hline \multirow{3}{*}{ Tratamento } & \multirow{3}{*}{$\mathbf{n}$} & \multicolumn{4}{|c|}{ PARTE DA PLANTA } \\
\hline & & \multicolumn{2}{|c|}{ Raízes } & \multicolumn{2}{|c|}{ Ramas } \\
\hline & & $\mathrm{x}$ & $\sigma$ & $\vec{x}$ & $\sigma$ \\
\hline No P1 K1 & 4 & 455,00 & 224,88 & 270,00 & 60,00 \\
\hline N1 Po K1 & 4 & 265,00 & 105,97 & 231,25 & 36,05 \\
\hline N1 P1 Ko & 4 & 940,00 & 191,13 & 761,25 & 125,98 \\
\hline N1 P1 K1 & 4 & $1.460,00$ & 206,40 & 483,75 & 101,78 \\
\hline N2 P1 K1 & 4 & $1.962,50$ & 137,69 & $1.037,50$ & 143,60 \\
\hline N1 P2 K1 & 4 & $1.750,00$ & 188,76 & 835,00 & 149,10 \\
\hline P1 K2 & 4 & $1.775,00$ & 206,32 & 757,00 & 50,58 \\
\hline
\end{tabular}

As fotografias anexas (Figs. 1 a 4) dão uma idéia do aspecto vegetativo das plantas aos três meses de idade.

3.1.1. Análise estatística. Foi feita separadamente, tomando-se N1 P1 K1 como testemunha. Considerou-se os tratamentos em que há ausência de um elemento, estudando-se a variação nas raízes e depois nas ramas; o mesmo se fez, a seguir, com os tratamentos em que há dose dupla do elemento. Os dados aparecem nos Quadros 2 a 5 . 


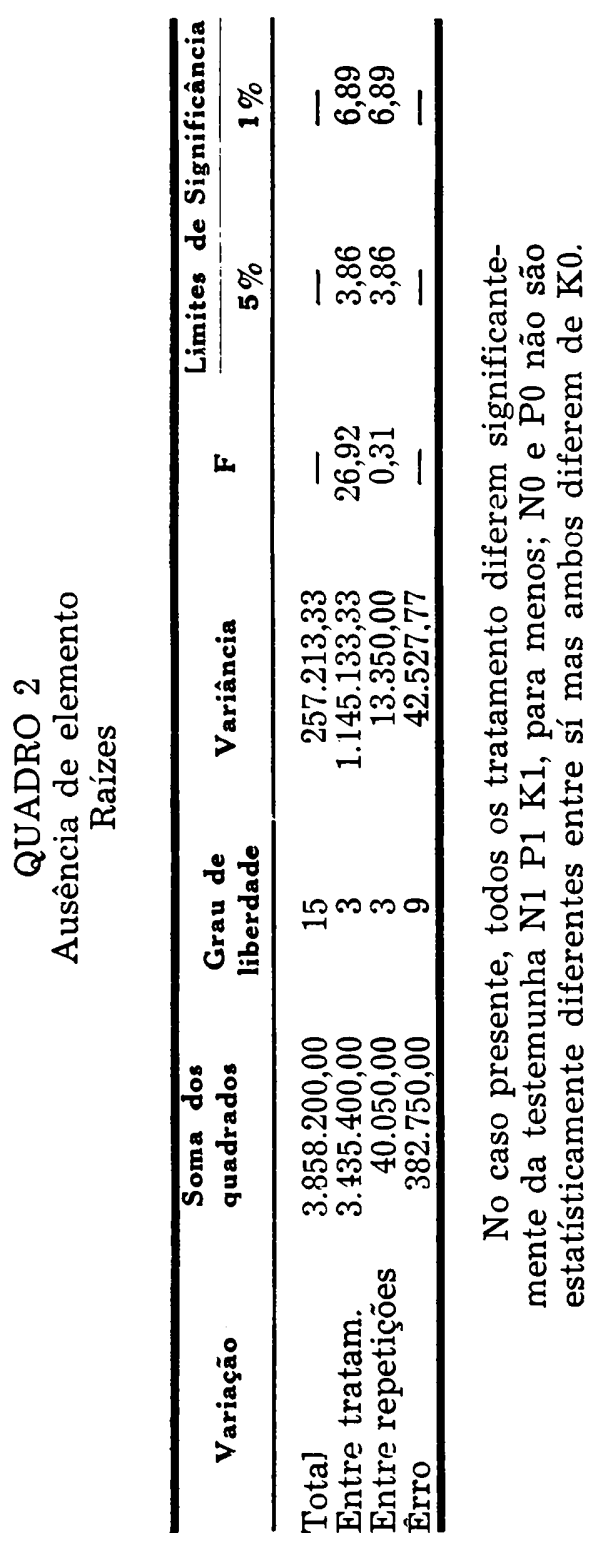




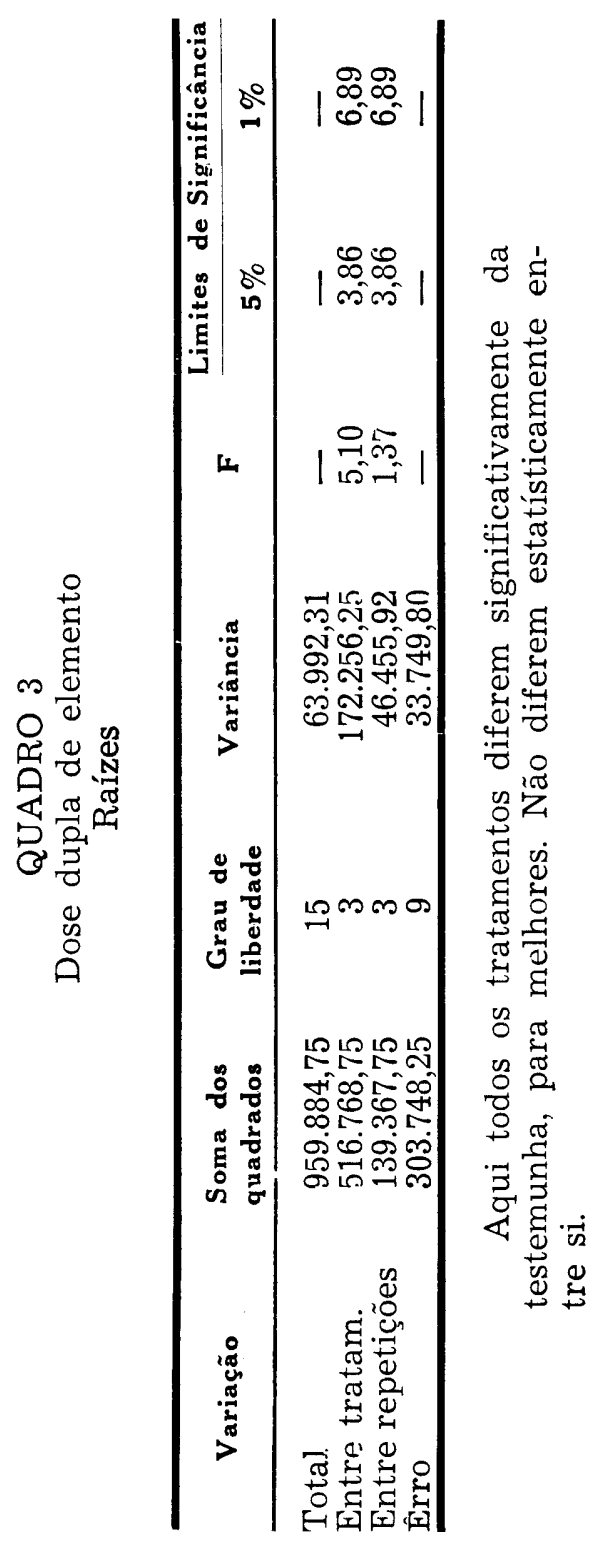




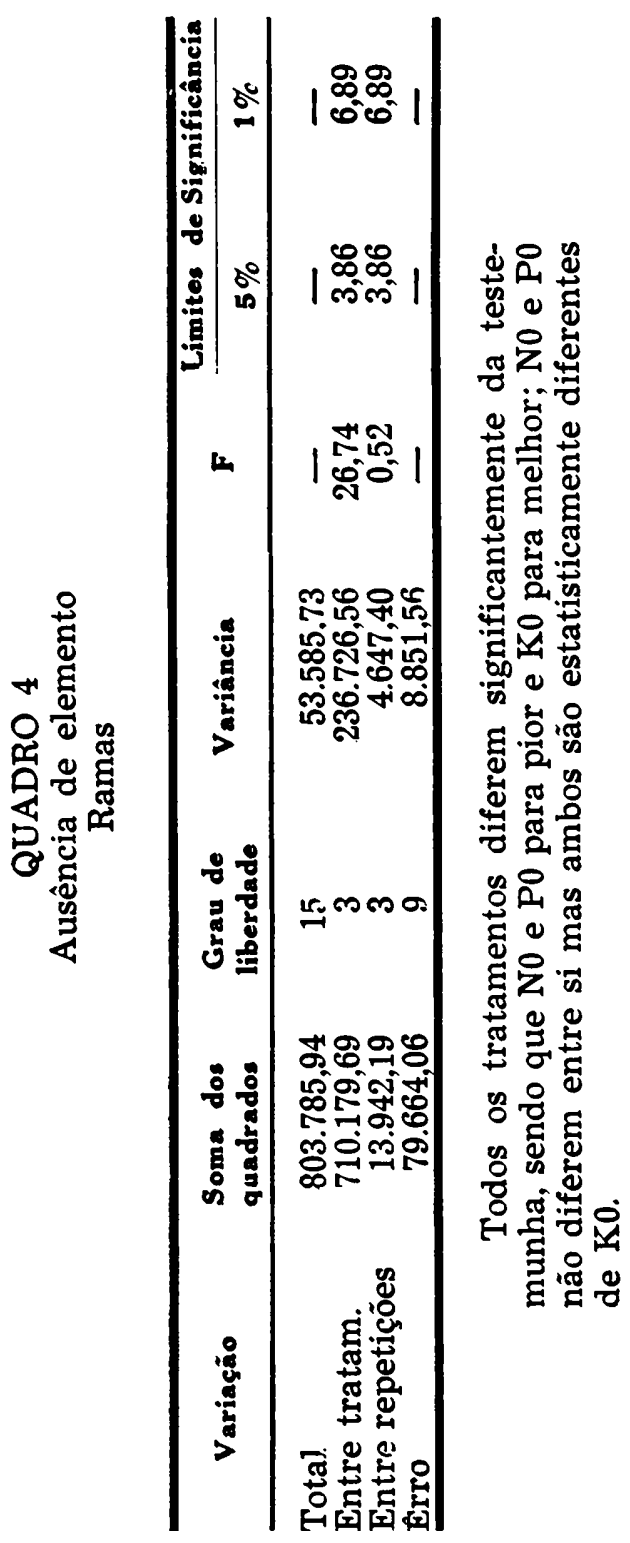




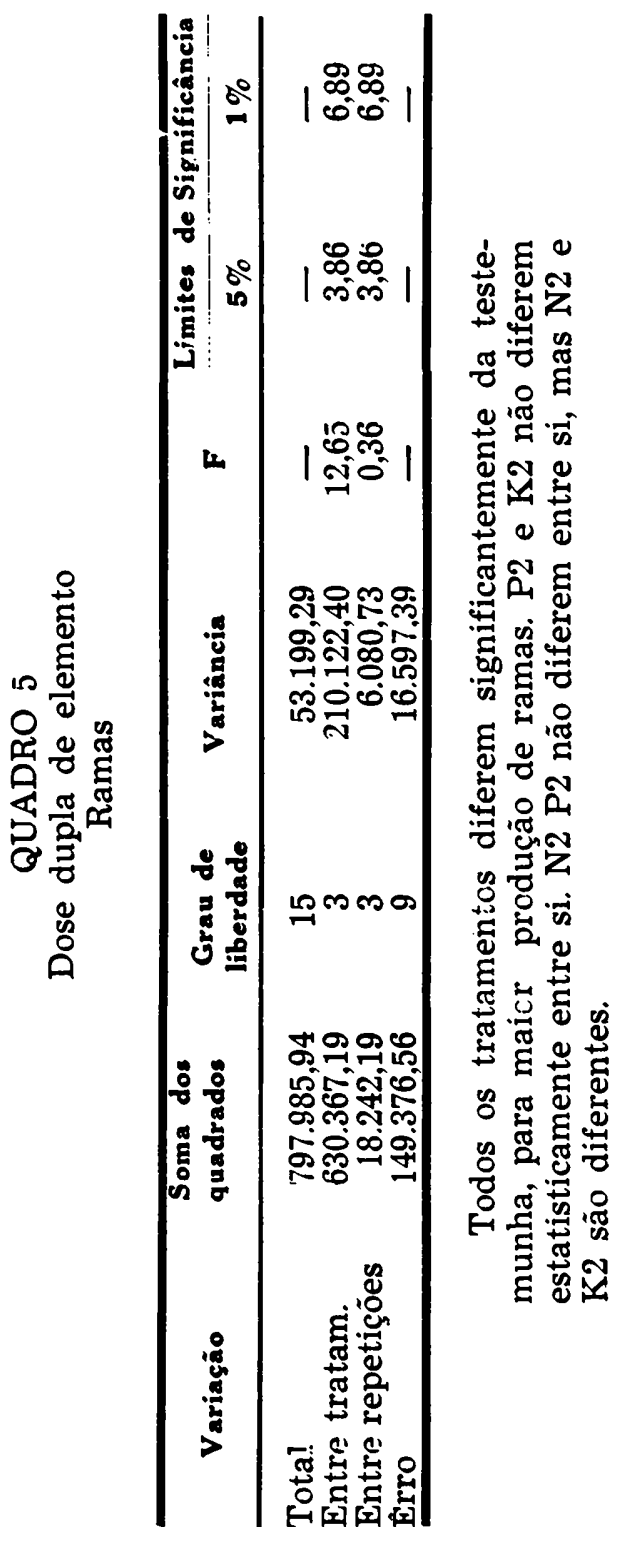


3.1.2. Discussão. Considerando-se as produções de raízes que são os dados que mais interessam ao presente ensaio - as diferenças entre tratamentos podem ser explicadas da seguinte maneira : dos três elementos estudados os que influem mais fortemente na produção da mandioca são o $\mathrm{P}$ e o $\mathrm{N}$; a necessidade do primeiro para a fosforilação das reservas de amido nos períodos iniciais de desenvolvimento é, por si só, suficiente para fazer entender a notável resposta observada; o nível ótimo de fósforo (ou seja, o mínimo, para citarmos Liebig) foi conseguido com a dose $\mathrm{P} 1$; uma vez estabelecido êsse ponto, a produção passa a ser controlada pelo nível de nitrogênio: admitindo-se isso, compreendemos logo o efeito favorável da dose dupla de nitrogênio, combinada às doses singelas de fósforo e potássio.

Resultado interessante foi encontrado com a ausência de $\mathrm{K}$, embora tivesse havidc redução na prcdução das raízes - como aliás, aconteceu na falta de $\mathrm{N}$ e $\mathrm{P}-$, houve aumento significante na produção das ramas. Embora ainda não se conheça exatamente tôdas as funções do potássio na planta, entre as reconhecidas há mais tempo, está o efeito desse elemento na transiocação dos carbohidratos (ver MEYER and ANDERSON, 1944, p. 423). A êsse respeito, COWIE (1951, pp. 50-51) cita ensaios em que plantas executaram todo o seu ciclo em solos de ficientes em potássio; o teor de carbohidratos nessas plantas era menor e os grãos se mostraram raquíticos por comparação com plantas recebendo quantidade adequada de potássio. Em vista disso, os presentes resultados se explicariam da seguinte maneira: como há dificuldade na translocação dos carbohidratos das folhas para as raizes, aqueles permaneceram na parte aérea e, como o fornecimento de nitrogênio era adequado, f $c$ ram consumidos na formação de mais material verde.

3.2. Resultados analíticos. Os dados resultantes das determinações químicas e também a análise estatística são dados a seguir. Com exceção da matéria sêca que só se acha expressa em porcentagem da substância original, os resultados são expressos em : porcentagem da substância sêca a $100-110^{\circ} \mathrm{C}$, porcentagem da substância original e em pêso total (gramas), por planta. $\mathrm{Na}$ análise estatistica se tomou o limite de 5 por cento. 
3.2.1. Matéria sêca.

\section{QUADRO 6}

Média da porcentagem de matéria sêca

\begin{tabular}{|c|c|c|c|c|c|c|c|}
\hline N1 P1 & $\mathbf{K 1}$ & No P1 & $\mathbf{K 1}$ & N1 Po & K1 & Ni P1 & $\mathbf{K o}_{\mathbf{0}}$ \\
\hline $\begin{array}{c}\text { N. Vaso } \\
] \\
2 . \\
3 \\
4 \\
\text { Médja }\end{array}$ & $\begin{array}{c}\% \\
40,65 \\
36,92 \\
39,28 \\
39,01 \\
38,96\end{array}$ & $\begin{array}{c}\text { N. Vaso } \\
5 \\
6 \\
7 \\
8 \\
\text { Médja }\end{array}$ & $\begin{array}{c}\% \\
40,55 \\
38,52 \\
38,27 \\
39,90 \\
39,31\end{array}$ & $\begin{array}{c}\text { N. Vaso } \\
9 \\
10 \\
11 \\
12 \\
\text { Média }\end{array}$ & $\begin{array}{c}\% \\
32,76 \\
34,27 \\
31,63 \\
32,48 \\
32,78\end{array}$ & $\begin{array}{c}\text { N. Vaso } \\
13 \\
14 \\
15 \\
16 \\
\text { Médja }\end{array}$ & $\begin{array}{c}\% \\
39,15 \\
36,65 \\
37,71 \\
36,44 \\
37,49\end{array}$ \\
\hline N2 $\quad$ P1 & K1 & $\begin{array}{ll}\text { N1 } & \text { P2 }\end{array}$ & K1 & N1 P1 & K2 & No Po & Ko \\
\hline $\begin{array}{c}\text { N. Vaso } \\
17 \\
18 \\
19 \\
20 \\
\text { Média }\end{array}$ & $\begin{array}{c}\% \\
31,54 \\
32,86 \\
34,46 \\
30,84 \\
32,42\end{array}$ & $\begin{array}{c}\text { N. Vaso } \\
21 \\
22 \\
23 \\
24 \\
\text { Média }\end{array}$ & $\begin{array}{c}\% \\
37,30 \\
39,29 \\
36,28 \\
38,98 \\
37,96\end{array}$ & $\begin{array}{c}\text { N. Vaso } \\
25 \\
26 \\
27 \\
28 \\
\text { Média }\end{array}$ & $\begin{array}{c}\% \\
34,07 \\
35,39 \\
38,84 \\
37,57 \\
36,47\end{array}$ & N. Vaso & 34,01 \\
\hline
\end{tabular}

A análise da variância mostrou o seguinte:

\section{QUADRO 7}

Análise da variância da matéria sêca

\begin{tabular}{|c|c|c|c|c|c|}
\hline Variação & $\begin{array}{l}\text { Soma dos } \\
\text { auadrados }\end{array}$ & $\begin{array}{l}\text { Grau de } \\
\text { liberdade }\end{array}$ & $\begin{array}{c}\text { Quadrados } \\
\text { médios }\end{array}$ & $\mathbf{F}$ & $\begin{array}{l}\text { Dif. minima } \\
\text { signif. }\end{array}$ \\
\hline $\begin{array}{l}\text { Total } \\
\text { Entre } \\
\text { Dentro }\end{array}$ & $\begin{array}{r}236,37 \\
189,91 \\
46,40\end{array}$ & $\begin{array}{r}27 \\
6 \\
21\end{array}$ & $\begin{array}{r}21,66 \\
2.21\end{array}$ & 14,33 & 2,186 \\
\hline
\end{tabular}

N2 P1 K1 e N1 P0 K1 não diferiram entre si, sendo ambos inferiores aos demais tratamentos. N1 P1 K1, N0 P1 K1, N1 P1 K0 e N1 P2 K1 não mostraram diferenças entre si. N1 P1 K2 foi inferior a N1 P1 K1 e a N0 P1 K1 e idêntico a N1 P2 K1 e a N1 P1 K0. 


\section{QUADRO 8}

Amido encontrado nos diferentes tratamentos

\begin{tabular}{|c|c|c|c|c|}
\hline Tratamento & Vaso & $\begin{array}{c}\mathrm{Na} \\
\text { Substâncih } \\
\begin{array}{c}\text { Sêca } \\
100-110^{\prime \prime} \mathrm{C} \\
\%\end{array}\end{array}$ & 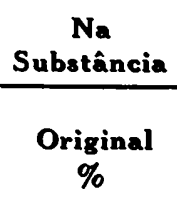 & $\begin{array}{c}\text { Total por } \\
\text { planta } \\
\text { g. }\end{array}$ \\
\hline N1 P1 K1 & $\begin{array}{r}1 \\
2 \\
3 \\
4 \\
\text { Média } \\
\end{array}$ & $\begin{array}{c}83,71 \\
81,63 \\
82,47 \\
80,43 \\
82,06 \\
\end{array}$ & $\begin{array}{r}34,03 \\
30,14 \\
32,39 \\
31,27 \\
31,98 \\
\end{array}$ & $\begin{array}{c}449,20 \\
524,44 \\
482,61 \\
404,67 \\
\quad 465,23 \\
\end{array}$ \\
\hline No P1 Kl & $\begin{array}{r}5 \\
6 \\
7 \\
8 \\
\text { Média } \\
\end{array}$ & $\begin{array}{r}83,70 \\
83,69 \\
83,63 \\
84,00 \\
\quad 83,75 \\
\end{array}$ & $\begin{array}{c}33,94 \\
32,24 \\
32,00 \\
33,52 \\
\quad 32,92 \\
\end{array}$ & $\begin{array}{c}251,16 \\
167,65 \\
73,60 \\
110,61 \\
\quad 150,75 \\
\end{array}$ \\
\hline N1 Po K1 & $\begin{array}{r}9 \\
10 \\
11 \\
12 \\
\text { Média }\end{array}$ & $\begin{array}{c}79,52 \\
78,58 \\
76,87 \\
78,29 \\
78,31 \\
\end{array}$ & $\begin{array}{l}26,05 \\
26,93 \\
24,31 \\
25,43 \\
25,68\end{array}$ & $\begin{array}{c}59,91 \\
105,03 \\
33,03 \\
76,29 \\
\quad 68,59\end{array}$ \\
\hline N1 P1 Ko & $\begin{array}{c}13 \\
14 \\
15 \\
16 \\
\text { Média }\end{array}$ & $\begin{array}{l}82,02 \\
78,84 \\
80,76 \\
80,46 \\
\quad 80.52 \\
\end{array}$ & $\begin{array}{c}32,11 \\
28,89 \\
30,45 \\
29,32 \\
\quad 30,19 \\
\end{array}$ & $\begin{array}{r}314,68 \\
196,45 \\
292,32 \\
334,25 \\
284,42 \\
\end{array}$ \\
\hline N2 P1 K1 & $\begin{array}{r}17 \\
18 \\
19 \\
20 \\
\text { Média } \\
\end{array}$ & $\begin{array}{c}75,13 \\
79,07 \\
77,32 \\
73,38 \\
76,22 \\
\end{array}$ & $\begin{array}{l}23,70 \\
25,98 \\
26,64 \\
22,63 \\
24,74 \\
\end{array}$ & $\begin{array}{l}497,70 \\
532,59 \\
479,52 \\
429,97 \\
\quad 484,91 \\
\end{array}$ \\
\hline N1 P2 K1 & $\begin{array}{r}21 \\
22 \\
23 \\
24 \\
\text { Média } \\
\end{array}$ & $\begin{array}{c}80,67 \\
79,19 \\
79,78 \\
80,04 \\
\quad 79,92 \\
\end{array}$ & $\begin{array}{l}30,09 \\
31,03 \\
28,94 \\
31,20 \\
30,31 \\
\end{array}$ & $\begin{array}{c}514,54 \\
546,13 \\
518,03 \\
542,88 \\
\quad 530,39 \\
\end{array}$ \\
\hline N1 P1 K2 & $\begin{array}{c}25 \\
26 \\
27 \\
28 \\
\text { Média } \\
\end{array}$ & $\begin{array}{c}78,59 \\
79,70 \\
78,49 \\
80,86 \\
79,41 \\
\end{array}$ & $\begin{array}{r}26,77 \\
28,28 \\
30,48 \\
30,38 \\
\quad 28,98\end{array}$ & $\begin{array}{c}495,24 \\
562,77 \\
457,20 \\
534,69 \\
512,47 \\
\end{array}$ \\
\hline
\end{tabular}




\subsubsection{Amido.}

A análise da variância vem a seguir

$$
\text { QUADRO } 9
$$

Análise da variância da quantidade total de amido (gramas)

\begin{tabular}{lrrrrrr}
\hline Variação & $\begin{array}{c}\text { Soma dos } \\
\text { quadrados }\end{array}$ & $\begin{array}{c}\text { Grau de } \\
\text { liberdade }\end{array}$ & $\begin{array}{c}\text { Quadrados } \\
\text { médios }\end{array}$ & F & $\begin{array}{c}\text { Dif. minima } \\
\text { signif. }\end{array}$ \\
\hline Total & $905.538,50$ & 27 & & & \\
Entre & $853.280,56$ & 6 & $142.213,42$ & 57,15 & 73,38 \\
Dentro & $52.257,95$ & 1 & $2.488,47$ & & \\
\hline
\end{tabular}

$\mathrm{N} 1 \mathrm{P} 0 \mathrm{~K} 1$ foi inferior a todos os demais. N1 P1 K0 foi superior a N0 P1 K1, porém inferior a N1 P1 K1, N2 P1 K1, N1 P1 K1 e N1 P2 K1, entre os quais nāo houve diferença significativa.

\subsubsection{Matéria mineral.}

A análise estatística referente aos dados da substância sêca a $100-110^{\circ} \mathrm{C}$ se acha no quadro seguinte.

$$
\text { QUADRO } 11
$$

Análise da variância da porcentagem de matéria mineral

\begin{tabular}{lrrrrr}
\hline Variação & $\begin{array}{r}\text { Soma dos } \\
\text { quadrados }\end{array}$ & $\begin{array}{c}\text { Grau de } \\
\text { liberdade }\end{array}$ & $\begin{array}{c}\text { Quadrados } \\
\text { médios }\end{array}$ & F & $\begin{array}{r}\text { Dif. minima } \\
\text { signif. }\end{array}$ \\
\hline Total & 7,36 & 27 & & & \\
Fintre & 6,52 & 6 & 1,08 & 27,18 & 0,293 \\
nontro & 0,84 & 1 & 0,04 & & \\
\hline
\end{tabular}

$\mathrm{O}$ tratamento $\mathrm{N} 1 \mathrm{P} 1 \mathrm{~K} 0$ foi inferior a todos os demais. N1 P2 K1 e N2 P1 K1 não diferiram entre si, nem de N1 P1 K1 e N1 P1 K2, sendo, porém, inferiores a N0 P1 K1. Este tratamento não diferiu de N1 P1 K2 e foi superior a N1 P1 K1. O tratamento N1 P0 K1 foi superior a todos os outros.

3.2.4. Matéria azotada. No quadro seguinte considera-se como matéria azotada o teor de nitrogênio multiplicado por 6,25 (Vêr Quadro n. 12.

A análise estatística referente aos dados da substância sêca a $100-110^{\circ} \mathrm{C}$ se acham no quadro seguinte 


\section{QUADRO 10}

Matéria mineral nos diferentes tratamentos

\begin{tabular}{|c|c|c|c|c|}
\hline \multirow[b]{2}{*}{ Tratamento } & \multirow[b]{2}{*}{ Vaso } & \multirow{2}{*}{$\begin{array}{c}\text { Na } \\
\text { Substância } \\
\text { Sêca } \\
\begin{array}{c}100-110^{\prime \prime C} \\
\%\end{array}\end{array}$} & \multirow{2}{*}{$\begin{array}{c}\mathrm{Na} \\
\text { Substância } \\
\begin{array}{c}\text { Original } \\
\%\end{array}\end{array}$} & \multirow{2}{*}{$\begin{array}{c}\text { Total por } \\
\text { planta } \\
\text { g. }\end{array}$} \\
\hline & & & & \\
\hline N1 P1 K1 & $\begin{array}{c}\text { Média } \\
1 \\
2 \\
3 \\
4 \\
\end{array}$ & $\begin{array}{c}2,51 \\
2,19 \\
2,74 \\
2,80 \\
2,56\end{array}$ & $\begin{array}{l}1,02 \\
0,81 \\
1,08 \\
1,09 \\
1,00 \\
\end{array}$ & $\begin{array}{c}13,56 \\
14,09 \\
16,09 \\
14,06 \\
14,45 \\
\end{array}$ \\
\hline No P1 K1 & $\begin{array}{r}5 \\
6 \\
7 \\
8 \\
\text { Média } \\
\end{array}$ & $\begin{array}{l}3,05 \\
3,28 \\
3,33 \\
2,89 \\
\quad 3,14\end{array}$ & $\begin{array}{l}1,23 \\
1,26 \\
1,27 \\
1,16 \\
\quad 1,23\end{array}$ & $\begin{array}{c}9,10 \\
6,55 \\
2,92 \\
5,28 \\
5,96\end{array}$ \\
\hline N1 Po K1 & $\begin{array}{c}9 \\
10 \\
11 \\
12 \\
\text { Média }\end{array}$ & $\begin{array}{l}3,52 \\
3,21 \\
3,83 \\
3,44 \\
-3,50 \\
\end{array}$ & $\begin{array}{l}1,15 \\
1,10 \\
1,21 \\
1,17 \\
\quad 1,16\end{array}$ & $\begin{array}{c}2,64 \\
4,29 \\
1,69 \\
3,51 \\
3,03\end{array}$ \\
\hline N1 P1 Ko & $\begin{array}{c}13 \\
14 \\
15 \\
16 \\
\text { Média }\end{array}$ & $\begin{array}{c}1,58 \\
1,89 \\
1,76 \\
2,08 \\
-\quad 1,83\end{array}$ & $\begin{array}{c}0,62 \\
0,69 \\
0,67 \\
0,75 \\
0,68 \\
\end{array}$ & $\begin{array}{r}6,08 \\
4,69 \\
6,43 \\
8,55 \\
6,44\end{array}$ \\
\hline N2 P1 K1 & $\begin{array}{c}17 \\
18 \\
19 \\
20 \\
\text { Média }\end{array}$ & $\begin{array}{l}2,72 \\
2,64 \\
2,46 \\
2,66 \\
2,62\end{array}$ & $\begin{array}{l}0,86 \\
0,87 \\
0,88 \\
0,82 \\
0,86\end{array}$ & $\begin{array}{c}18,06 \\
17,83 \\
15,84 \\
15,58 \\
16,83\end{array}$ \\
\hline N1 P2 K1 & $\begin{array}{c}21 \\
22 \\
23 \\
24 \\
\text { Média }\end{array}$ & $\begin{array}{l}2,77 \\
2,56 \\
2,94 \\
2,82 \\
\quad 2,77\end{array}$ & $\begin{array}{l}1,03 \\
1,00 \\
1,06 \\
1,10 \\
1,05\end{array}$ & $\begin{array}{c}17,61 \\
17,60 \\
18,97 \\
19,14 \\
\quad 18.33\end{array}$ \\
\hline N1 P1 K2 & $\begin{array}{c}25 \\
26 \\
27 \\
28 \\
\text { Média } \\
\end{array}$ & $\begin{array}{l}3,02 \\
2,80 \\
2,76 \\
2,89 \\
2,87\end{array}$ & $\begin{array}{l}1,03 \\
0,99 \\
1,07 \\
1,08 \\
1,04\end{array}$ & $\begin{array}{r}19,05 \\
19,70 \\
16,05 \\
19,01 \\
18,45\end{array}$ \\
\hline
\end{tabular}


QUADRO 12

Matéria azotada em diferentes tratamentos

\begin{tabular}{|c|c|c|c|c|}
\hline Tratamento & Vaso & $\frac{\begin{array}{c}\text { Na } \\
\text { Substância }\end{array}}{\begin{array}{c}\text { Séca } \\
100-110^{\prime \prime C} \\
\%\end{array}}$ & $\frac{\begin{array}{c}\text { Na } \\
\text { Substância }\end{array}}{\begin{array}{c}\text { Original } \\
\%\end{array}}$ & $\begin{array}{c}\text { Total por } \\
\text { planta } \\
8 .\end{array}$ \\
\hline N1 P1 K1 & $\begin{array}{c}1 \\
2 \\
3 \\
4 \\
\text { Média }\end{array}$ & $\begin{array}{l}2,52 \\
2,85 \\
3,02 \\
3,26 \\
2,91\end{array}$ & $\begin{array}{l}1,02 \\
1,06 \\
1,19 \\
1,27 \\
\quad 1,13\end{array}$ & $\begin{array}{l}13,47 \\
18,44 \\
17,73 \\
16,38 \\
\quad 16,50 \\
\end{array}$ \\
\hline No $\mathrm{P} 1 \mathrm{~K} 1$ & $\begin{array}{r}5 \\
6 \\
7 \\
8 \\
8 \\
\text { Média } \\
\end{array}$ & $\begin{array}{c}1,50 \\
1,33 \\
1,65 \\
1,84 \\
1,58\end{array}$ & $\begin{array}{c}0,61 \\
0,51 \\
0,63 \\
0,73 \\
\quad 0,62 \\
\end{array}$ & $\begin{array}{c}4,51 \\
2,65 \\
9]^{{ }^{\prime}} I \\
2,41 \\
2,75\end{array}$ \\
\hline N1 Po K1 & $\begin{array}{c}9 \\
10 \\
11 \\
12 \\
\text { Média }\end{array}$ & $\begin{array}{l}3,91 \\
4,56 \\
4,91 \\
4,40 \\
\quad 4,44 \\
\end{array}$ & $\begin{array}{c}1,18 \\
1,56 \\
1,55 \\
1,43 \\
1,43\end{array}$ & $\begin{array}{r}2,71 \\
6,08 \\
2,17 \\
4,29 \\
\quad 3,81 \\
\end{array}$ \\
\hline N1 P1 Ko & $\begin{array}{r}13 \\
14 \\
15 \\
16 \\
\text { Média } \\
\end{array}$ & $\begin{array}{c}4,67 \\
2,92 \\
3,85 \\
3,40 \\
\quad 3,71\end{array}$ & $\begin{array}{c}1,83 \\
1,00 \\
1,45 \\
1,24 \\
\quad 1,40 \\
\end{array}$ & $\begin{array}{c}17,93 \\
7,28 \\
13,92 \\
14,40 \\
13,32 \\
\end{array}$ \\
\hline N2 P1 K1 & $\begin{array}{r}17 \\
18 \\
19 \\
20 \\
\text { Médio }\end{array}$ & $\begin{array}{l}5,55 \\
4,85 \\
4,54 \\
5,61 \\
5,14 \\
\end{array}$ & $\begin{array}{c}1,75 \\
1,5 y \\
1,56 \\
1,73 \\
1,66 \\
\end{array}$ & $\begin{array}{l}36,75 \\
35,59 \\
28,08 \\
32,87 \\
\quad 32,57 \\
\end{array}$ \\
\hline N1 P2 K1 & $\begin{array}{r}21 \\
22 \\
23 \\
24 \\
\text { Média } \\
\end{array}$ & $\begin{array}{r}3,01 \\
3,44 \\
3,45 \\
3,01 \\
3,23 \\
\end{array}$ & $\begin{array}{l}1,12 \\
1,35 \\
1,25 \\
1,17 \\
1,22\end{array}$ & $\begin{array}{l}19,15 \\
23,76 \\
22,37 \\
20,36 \\
21,41\end{array}$ \\
\hline N1 P1 K2 & $\begin{array}{c}25 \\
26 \\
27 \\
28 \\
\text { Médio } \\
\end{array}$ & $\begin{array}{l}3,27 \\
3,39 \\
3,17 \\
3,01 \\
3,21\end{array}$ & $\begin{array}{l}1,11 \\
1,20 \\
1,23 \\
1,13 \\
1,17\end{array}$ & $\begin{array}{l}20,53 \\
23,88 \\
18,45 \\
19,89 \\
\quad 20,69\end{array}$ \\
\hline
\end{tabular}




\section{QUADRO 13}

Análise da variância da matéria azotada

\begin{tabular}{lrrrrr}
\hline Variação & $\begin{array}{r}\text { Soma dos } \\
\text { quadrados }\end{array}$ & $\begin{array}{c}\text { Grau de } \\
\text { liberdade }\end{array}$ & $\begin{array}{c}\text { Quadrados } \\
\text { médioz }\end{array}$ & F & $\begin{array}{r}\text { Dif. mínima } \\
\text { signif. }\end{array}$ \\
\hline Total & 34,90 & 27 & & & \\
Entre & 31,19 & 6 & 5,20 & 24,92 & 0,618 \\
Dentro & 3,71 & 21 & 0,17 & & \\
\hline
\end{tabular}

Todos os tratamentos foram inferiores a N2 P1 K1 e superiores a N0 P1 K1; não houve diferenças entre N1 P1 K0, N1 P2 K1 e N1 P1 K2 que foram inferiores a N1 P0 K1. A adubação completa, N1 P1 K1, foi inferior a N1 P1 K0 e igual a N1 P2 K1 e N1 P1 K2.

3.2.5. Discussão. Percorrendo-se os resultados analíticos, verifica-se logo que do ponto de vista econômico os dados mais interessantes são os que aparecem em 3.2.2. Há uma variação surpreendente nos teores porcentual e total de amido quando se considera principalmente a atuação do nitrogênio e do fósforo. Aliás, o comportamento do fósforo no que se refere à formação do amido acompanhou bem o que se verificou nos dados de produção (vêr 3.1.). A ausência de fósforo provocou um decréscimo considerável na produção e uma queda na quantidade de amido, tanto porcentual como total. O papel fundamental do fósforo no metabolismo dos carbohidratos através das fosforilações explica suficientemenie o resultado visto. Quanto à ação do nitrogênio, compare-se os dados de produção com os resultados que aparecem no Quadro 8 (vêr 3.2.2.) : a dose dupla de nitrogênio garantiu a maior produção de raízes obtida; entretanto, tal produção mais elevada não se traduz em benefício alguin: de fato, o Quadro 8 mostra logo que o aumento na dose de nitrogênio acarretou uma queda na porcentagem de amido na substância original de pràticamente 32 por cento para 24 por cento; em consequência, a quantidade total de amido obtida não diferiu significantemente daquela conseguida com a dose simples de nitrogênio. A queda porcentual do amido na substância original encontra explicação nos dados do Quadro 12 (vêr 3.2.4.) onde se vê que: devido ao aumento na proporção de nitrogênio na relação deu-se uma elevação de quase 50 por cento no teor de matéria azotada; é lícito, então admitir que, quando se elevou a quantidade de $\mathrm{N}$ fornecida às plantas, uma 
porção considerável de carbohidratos, em lugar de se polimerizar a amido, se combinou ao nitrogênio disponivel convertendo-se em proteina ou outras substâncias nitrogenadas com esqueleto carbônico.

\section{4 - RESUMO E CONCLUSŐES}

4.1. O presente trabalho foi conduzido para estudar o efeito da adubação mineral na produção da mandioca, bem como na composição das raízes. A variedade "Branca de Sta. Catarina" foi cultivada em vasos cheios de areia lavada aos quais se adicionou vários sais de modo a ter os seguintes tratamentos: N0 P0 K0, N0 P1 K1, N1 P0 K1, N1 P1 K0, N2 P1 K1, N1 P2 K1 e N1 P1 K2 onde o índice significa a dose relativa do elemento nutritivo considerado. Cada tratamento foi feito com quatro repetições. As doses usadas foram as seguintes: $\mathrm{N} 1=35$ gramas de nitrato de amônio por vaso com 60 quilos de areia; $\mathrm{P} 1=35$ gramas de fosfato monocálcico e $\mathrm{K} 1=28$ gramas de sulfato de potássio. Além disso, todos os vasos receberam 26 gramas de sulfato de magnésio e micronutrientes em doses semanais, na forma e proporções indicadas por HOAGLAND e ARNON (1939).

4.2. No que se refere à produção (tanto de ramas como de raízes) os seguintes pontos devem ser considerados:

4.2.1. O efeito mais saliente na produção foi devido à omissão do $\mathrm{P}$ e do $\mathrm{N}$ na adubação; com isso foram obtidas as colheitas mais baixas do ensaio; a necessidade do primeiro para a fosforilação das reservas amiláceas deve explicar o resultado que se observou.

4.2.2. O fósforo e o nitrogênio, nas condições do ensaio, foram os elementos mais importantes para a mandioca; o efeito do potássio na produção de raízes foi muito menos acentuado do que aquele dos dois macronutrientes citados; é interessante mencionar que em ausência de potássio, embora a produção de raízes diminuisse, a de ramas cresceu; à vista da literatura sôbre os possiveis papéis do potássio no metabolismo dos carbohidratos, é razoável admitir que, a falta de potássio impedindo a translocaçăo de açúcares, éstes tivessem sido consumidos na produção de ramas. 
4.3 A análise química das raízes mostrou alguns resultados dignos de menção:

4.3.1. A falta de fósforo produziu a redução mais drástica no teor de amido das raízes; enquanto no tratamento N1 P1 K1 se tinha 32 por cento de amido, no tratamento N1 P0 K1, o teor encontrado foi de 25 por cento; isto se explica provàvelmente, pela necessidade de $\mathrm{P}$ para a síntese enzimática dêsse polisacarídeo; convém notar que o decréscimo na porcentagem de amido esteve associado com a considerável queda na produção consequente da falta do elemento $\mathrm{P}$ do meio nutritivo.

4.3.2. Com a dose dupla de nitrogênio no tratamento N2 P1 K1 se obteve a maior produção observada; porém tal aumento na colheita não se traduziu em nenhuma vantagem industrial pela seguinte razão : enquanto o tratamento N1 P1 K1 produziu 32 por cento de amido, a duplificação da dose de nitrogênio fez aquela porcentagem baixar para 24; e o que é importante : o aumento total na quantidade de amido não difere estatisticamente daquela obtida com a dose simples de nitrogênio; o que se observou aqui com $\mathrm{N}$ e amido é muito semelhante ao que ocorre com $\mathrm{N}$ e sacarose em beterraba e cana; trata-se de um aspecto bioquímico do efeito da adubação muito interessante: aumentando-se a quantidade de nitrogênio disponível em lugar de os carbohidratos se polimerizarem a amido, se combinam com aquele para produzir substância protêica; houve de fato, uma elevação no teor de proteina de 2,91 a 5,14 por cento.

\section{5 - SUMMARY}

5.1. The present work was carried out to study the effects of mineral nutrients in the yield as well as in the composition of cassava roots. The variety "Branca de Sta. Catarina" was grown by the sand culture method, the following treatments being used: N0 P0 K0, N0 P1 K1, N1 P0 K1, N2 P1 K0, N2 P1 K1, N1 P2 K1, and N1 P1 K2, where the figures 0, 1, and 2 denote the relative proportion of a given element. The nutrients were given as follows: $\mathrm{N}=35$ grams of ammonium nitrate per pot loaded with 120 pounds of washed sand; $P 1=35$ 
grams of monocalcium phosphate; $K 1=28$ grams of sulfate of potash. Besides those fertilizers, each pot received 26 grams of magnesium sulfate and weekly doses of micronutrients as indicated by HOAGLAND and ARNON (1939). To apply the macronutrients the total doses were divided in three parts evenly distributed during the life cycle of cassava.

5.2. As far yield of roots and foliage are concerned, there are a few points to be considered :

5.2.1. the most striking effect on yield was verified when $P$ was omitted from the fertilization; this treatment gave the poorest yields of the whole experiment; the need of that element for the phosphorylation of the starchy reserves explains such result;

5.2.2. phosphorus and nitrogen, under the experimental conditions, showed to be the most important nutrients for cassava; the effect of potassium in the weight of the roots produced was much less marked; it is noteworthy to mention, that in absence of potassium, the roots yield decreased whereas the foliage increased; as potassium is essential for the translocation of carbohydrates it is reasonable to admit that sugars produced in the leaves instead of going down and accumulate as starch in the roots were consumed in the production of more green matter.

5.3. Chemical analyses of roots revealed the following interesting points :

5.3.1. the lack of phosphorus brought about the most drastic reduction in the starch content of the roots; while the treatment N1 P1 K1 gave 32 per cent of starch, with N1 P0 K1 the amount found was 25 per cent; this result can be explained by the requirement of $\mathrm{P}$ for the enzymatic synthesis of starch; it has to be mentioned that the decrease in the starch content was associated with the remarkable drop in yield observed when $P$ was omitted from the nutrient medium;

5.3.2. the double dosis of nitrogen in the treatment N2 P1 K1, gave the highest yields; however the increase in yield did not 
produce any industrial gain : whereas the treatment N1 P1 K1 gave 32 per cent of starch, by raising the $\mathrm{N}$ level to $\mathrm{N2}$, the starch content fell to 24 per cent; now, considering the total amount of starch present in the roots, one can see, that the increase in roots yield did not compensate for the marked decrease in the starch content; that is, the amount of starch obtained with N1 P1 K1 does not differ statistically from the quantity obtained with N2 P1 K1; as far we know facts similar to this had been observed in sugar beets and sugar cane, as a result of the interaction between nitrogen and sugar produced; the biochemical aspect of the problem is very interesting : by raising the amount of assimilable nitrogen, instead of the carbohydrates polymerize to starch, they do combine to the amino groups to give proteinaceous materials; actually, it did happen that the protein content increased from 2.91 to 5.14 per cent.

\section{6 - LITERATURA CITADA}

COWIE, G. A. 1951 Em "Potash", Edward Arnold and Co., London.

EWERS, E. 1908 Uber polarimetrische starkebestimmung. Chemiker Zeit. 32: 996-997.

GOMES, F. PIMENTEL e E. MALAVOLTA. 1949 Aspectos matemáticos e estatísticos da lei de Mitscherlich. An. E. S. A. "Luiz de Queiroz", U.S.P. 6: 193-229.

GROSSMAN, J. 1946 A cultura da mandioca no Rio Grande do Sul. Rev. Fac. Agron. (Montevideo) 42: 1-33.

HOAGLAND, D. R. and D. I. ARNON. 1939 The water culture method for growing plants without soil. Univ. of California Agr. Expt. Sta. (Berkeley, Calif.) Circ. 347.

KJELDAHL, J. 1883 Neue Methode zur Bestimmung der Sticksfoffs in organischen Korpen. Z. anal. Chem. 22: 366-382. 
MALA VOLTA, E., T. COURY, E. A. GRANER, J. A. DE C. PACHECO, M. O. C. DO BRASIL SOBR. 1953 Adubação da mandioca (Manihot utilissima Pohl) I. Ensaio em areia lavada (Nota prévia). An. E. S. A. "Luiz de Queiroz", U.S.P.

MEYER, B. S. and D. B. ANDERSON. 1944 Em "Plant Physiology", Fourth Printing, D. Van Nostrand Co., Inc., New York.

NORMANHA, E. S. e A. S. PEREIRA. 1950 Aspectos agronômicos da cultura da mandioca (Manihot utilissima Pohl.) Bragantia 10: 179-202.

RIECHELMANN. 1921 Zur Bestimmung der Starke in Manihotmehl. Zeitsc. f. offentliche Chem. 27: 5.

WALTON, R. P. 1928 Em "A comprehensive survey of starch chemistry", vol. 1, The Chemical Catalog Co. 